\title{
NONLINEAR-COUPLED FREE OSCILLATION ANALYSIS OF SUSPENSION BRIDGES
}

\author{
By Hiroki YAMAGUCHI* and Kazushige ARAKAWA**
}

\begin{abstract}
A method of analysis is developed for coupled free oscillations of suspension bridges in three dimensions. The method, which is based on a finite-element approach, takes into account the nonlinear coupling effects by means of considering the finite displacements of the main cables and the hangers. The accuracy and efficiency of the proposed method are demonstrated by comparing the computed results with the experimental values and the previously computed values reported in Refs. 18, 19. The nonlinear-coupled characteristics in the torsion-dominant first mode are also investigated for a suspension bridge under construction and a suspension bridge deformed by wind forces.
\end{abstract}

Keyword: suspension bridge, nonlinear, coupled oscillation, FEM.

\section{INTRODUCTION}

Suspension bridges are so flexible, compared with other types of bridges, that severe oscillations can occur easily due to various dynamic loads, especially due to wind forces. The great span lengths of recent suspension bridges make their aerodynamic behaviors more important problems. The torsional flutter is one of the most serious phenomena of wind-induced vibrations of suspension bridges. Consequently, torsional-oscillation analysis must be performed prior to the standard wind tunnel tests in order to determine natural frequencies and associated mode shapes.

Free torsional oscillations of suspension bridges are rarely pure torsional motions of the stiffening structure (truss, box girder, etc.) but they are generally coupled with lateral movements ${ }^{1}$. For suspension' bridges deformed laterally by wind forces, vertical motions are also coupled with the torsion-dominant oscillations ${ }^{2), 3)}$. This is caused by the structure's geometrical nonlinearity which is due to the inherent flexibility. These translational motions coupled with the torsional oscillation may play an important role particularly when the aerodynamic stability for the torsional flutter is considered, because such coupled motions are reflected directly on the aerodynamic forces acting on the suspension bridge. In accordance with this, it becomes important to analyze realistically the coupled free oscillations of suspension bridges in three dimensions.

The aerodynamic stability of a suspension bridge under construction becomes also very important with the long construction period for long-span-length bridges. The dynamic behavior of the suspension bridge under construction is much more complicated because of the increase of its flexibility; this means the role played by the geometrical nonlinearity also increases ${ }^{4}$.

* Member of JSCE, Dr. Eng., Asst. Professor of Foundation Eng., Saitama Univ. (255 Shimo-ohkubo, Urawa, Saitama)

** Member of JSCE, M. Eng., Japan Highway Public Corporation (Formerly Graduate Student, Saitama Univ.) 
Many investigations have been made to compute the natural frequencies and modes of free vibrations of suspension bridges ${ }^{5 / 14)}$. Especially Abdel-Ghaffar's series of papers ${ }^{9 / 14)}$ dealing with the dynamic analysis of suspension bridges in which effective analytical procedures were developed for various types of vibrations including nonlinear free oscillations. It was suggested in his papers that the vibrational modes of suspension bridge are generally three-dimensional modes and that the coupling of lateral, torsional and vertical vibrations should be considered in the analysis, but the coupling effects were neglected, in some of his papers, and each oscillation was treated independently because of the complexity in the calculation and of their small effects on linear oscillations. Only the nonlinear coupled vertical-torsional vibrations of suspension bridges were analyzed, in some of his papers, for the large amplitude free oscillations ${ }^{13)}$.14). $^{\text {. }}$

Although some general-purpose computer programs for the analysis of structure, such as SAP 4, FEAP, etc., may be also applicable to analyze the free oscillations of suspension bridges, those programs have a disadvantage with respect to the efficiency in the sense that some degrees of freedom (DOF), which are not necessary for suspension bridges, should be considered ${ }^{15)}$.

In the present paper, an efficient method is developed to analyze the coupled free oscillations of suspension bridges in three dimensions. The method, which is based on the same finite-element-approach as Abdel-Ghaffar's method, takes into account the nonlinear coupling effects by means of considering the finite displacements of cables and hangers. The role played by the hangers, which governs the coupling behavior, is considered strictly; the small motion of a hanger, which is assumed to be inextensible to reduce the total number of $\mathrm{DOF}$, is considered from an equilibrium position in order to obtain the tangential stiffness matrix of hanger member.

An incremental method is utilized to analyze the nonlinear static deformation of a suspension bridge subjected to external forces such as wind loads. In addition linear free oscillations of the deformed suspension bridge are analyzed to determine the natural frequencies and the nonlinear-coupled modes of free oscillations. The accuracy and efficiency of the proposed method are demonstrated by comparing the computed results with the experimental values ${ }^{18)}$ and some previously computed results using the modified SAP 4 program ${ }^{19)}$. Finally the nonlinear-coupled characteristics of the torsion-dominant first mode are investigated for a suspension bridge under construction and for a suspension bridge deformed by wind forces.

\section{ANALYTICAL MODEL OF SUSPENSION BRIDGE}

\section{(1) Assumptions}

The following assumptions are introduced in the analysis.

1) The stiffening structure of the suspension bridge is treated as a Bernoulli-Euler beam. The axial displacement of the girder is neglected and other displacements are assumed to be sufficiently small.

2) The main cables are assumed to be perfectly flexible and the geometrical nonlinearity of such cables is taken into consideration.

3) The hangers are assumed to be massless and inextensible strings and their rigid-body movements are finite.

The static lateral-deflection of the bridge deck induced by extreme wind forces, such as the design or critical wind speed, is at most a few percent of the span length. This fact allows one to assume a linear beam theory for the stiffening structure.

The nonlinearity of a suspension bridge is considered to be caused mainly by the geometrical nonlinearity of the main cables and by the large motions of the inextensible hangers which act as linkages between the stiffening structure and the main cables (Fig. 1). These types of nonlinearity can be treated by the assumptions 2) and 3).

\section{(2) Finite-element discretization and DOF}

The suspension bridge is subdivided into a discrete system consisting of suspension-bridge elements as 


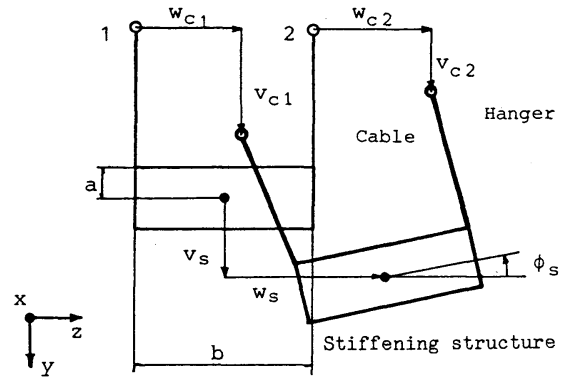

Fig. 1 Deformations of a cross section.
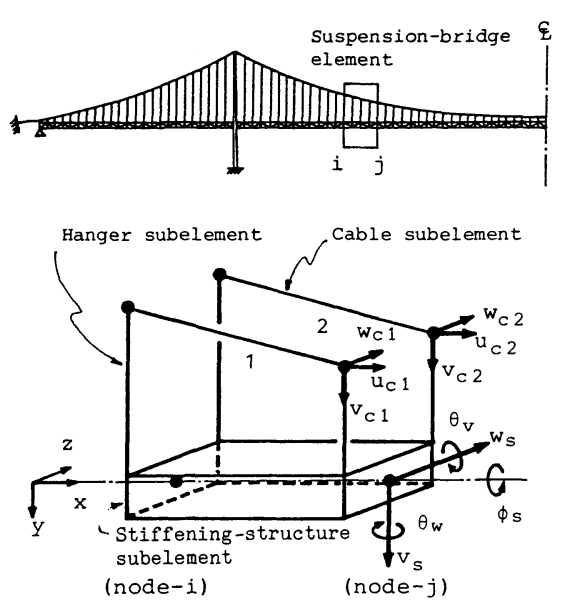

Fig. 2 Suspension-bridge element.

shown in Fig. 2. Each suspension-bridge element is composed of subelements of a stiffening structure, two main cables and four hangers. The stiffening-structure subelement is represented by a beam element and the DOF at each node is five ; two components of deflection $v_{s}, w_{s}$, two corresponding rotations $\theta_{v}, \theta_{w}$ and one torsional angle $\phi_{s}$. The main-cable subelement is treated by a nonlinear cable element which is described later on and the DOF at a nodal point of the cable element is three; $u_{c}, v_{c}, w_{c}$. As for the hangers, their role is to restrain the relative movements of the beam element and the cable elements. The compatibility condition is imposed through one relationship between the beam displacements and the cable displacements for each hanger. The total number of DOF per one cross section (node) is, therefore, nine $(5+2 \times 3-2 \times 1=9)$ which is less than the half the number of DOF in any ordinary finite-element model ${ }^{2}$.

\section{STIFFNESS AND MASS MATRICES FOR EACH SUBELEMENT}

\section{(1) Stiffening structure-Bernoulli-Euler beam element}

The stiffness matrix $\left[k_{s}\right]$ and the mass matrix $\left[m_{s}\right]$ for the beam element are the general matrices for the ordinary beam element ${ }^{16)}$. The corresponding displacement vector $\left\{q_{s}\right\}$ is given by

$$
\left\{q_{s}\right\}=\left[\begin{array}{llllllllll}
v_{s i} & \theta_{v i} & w_{s i} & \theta_{w i} & \phi_{s i} & v_{s j} & \theta_{v j} & w_{s j} & \theta_{w j} & \phi_{s j}
\end{array}\right]^{T}
$$

(2) Main cables nonlinear cable element

The initial equilibrium shape $\left(x, y_{c}, 0\right)$, which is assumed to be a parabola due to dead loads uniformly distributed along the bridge axis $x$, is chosen as a reference state. Introducing the curvilinear coordinate, $s$, which is defined along the actual length of the parabola, the unit elongation $\varepsilon_{c}$ can be written as

$$
\varepsilon_{c}=\left\{u_{c}^{\prime}+y_{c}^{\prime} v_{c}^{\prime}+\frac{1}{2}\left(u_{c}^{\prime 2}+v_{c}^{\prime 2}+w_{c}^{\prime 2}\right)\right\}\left(\frac{d x}{d s}\right)^{2} \text {. }
$$

where ()$^{\prime}$ denotes the differentiation with respect to $x$.

The strain energy $V_{c}$ of the main cable is represented by

$$
V_{c}=H_{w} \int_{0}^{l} \varepsilon_{c} d x+\frac{1}{2} E A \int_{0}^{l} \varepsilon_{c}^{2}\left(\frac{d s}{d x}\right) d x
$$

in which $H_{w}$ is the horizontal component of the initial cable-tension, EA is the axial rigidity of cable and $l$ is the length of the suspension-bridge element.

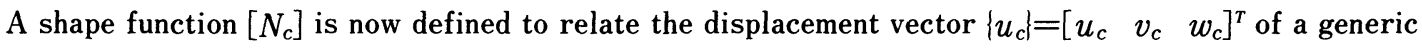
point on the cable element to the nodal displacement vector $\left\{q_{c}\right\}=\left[\begin{array}{llllll}u_{c i} & v_{c i} & w_{c i} & u_{c j} & v_{c j} & w_{c j}\end{array}\right]^{T}$ as

$\left\{u_{c}\right\}=\left[N_{c}\right]\left\{q_{c}\right\}$

In this paper, a linear displacement field is used for the cable subelement.

Combining Eqs. (2), (3) and (4), and taking the first variation of the potential energy, one can 
obtain the so-called elastogeometric nonlinear matrix ${ }^{17)}$ for the cable subelement as follows:

$$
\left[k_{c}^{E}\right]=\left[\begin{array}{rr}
{[k]} & -[k] \\
-[k] & {[k]}
\end{array}\right] ; \text { with }[k]=\left[\begin{array}{lll}
k_{c 11} & k_{c 12} & k_{c 13} \\
& k_{c 22} & k_{c 23} \\
\text { sym. } & & k_{c 33}
\end{array}\right]
$$

where

$$
\begin{aligned}
& k_{c 11}=\frac{H_{w}}{l}+\frac{E A}{l}\left(\frac{d x}{d s}\right)^{3}\left[1-\frac{1}{2 l}\left\{3\left(u_{c i}-u_{c j}\right)+y^{\prime}\left(v_{c i}-v_{c j}\right)\right\}+\frac{1}{4 l^{2}}\left\{\left(u_{c i}-u_{c j}\right)^{2}+\Delta^{2}\right\}\right] \\
& k_{c 22}=\frac{H_{w}}{l}+\frac{E A}{l}\left(\frac{d x}{d s}\right)^{3}\left[\left(y^{\prime}\right)^{2}-\frac{1}{2 l}\left\{\left(u_{c i}-u_{c j}\right)+3 y^{\prime}\left(v_{c i}-v_{c j}\right)\right\}+\frac{1}{4 l^{2}}\left\{\left(v_{c i}-v_{c j}\right)^{2}+\Delta^{2}\right\}\right] \\
& k_{c 33}=\frac{H_{w}}{l}+\frac{E A}{l}\left(\frac{d x}{d s}\right)^{3}\left[-\frac{1}{2 l}\left\{\left(u_{c i}-u_{c j}\right)+y^{\prime}\left(v_{c i}-v_{c j}\right)\right\}+\frac{1}{4 l^{2}}\left\{\left(w_{c i}-w_{c j}\right)^{2}+\Delta^{2}\right\}\right] \\
& k_{c 12}=\frac{E A}{l}\left(\frac{d x}{d s}\right)^{3}\left[y^{\prime}-\frac{1}{2 l}\left\{y^{\prime}\left(u_{c i}-u_{c j}\right)+\left(v_{c i}-v_{c j}\right)\right\}+\frac{1}{4 l^{2}}\left(u_{c i}-u_{c j}\right)\left(v_{c i}-v_{c j}\right)\right] \\
& k_{c 13}=\frac{E A}{l}\left(\frac{d x}{d s}\right)^{3}\left[-\frac{1}{2 l}\left(w_{c i}-w_{c j}\right)+\frac{1}{4 l^{2}}\left(u_{c i}-u_{c j}\right)\left(w_{c i}-w_{c j}\right)\right] \\
& k_{c 23}=\frac{E A}{l}\left(\frac{d x}{d s}\right)^{3}\left[-\frac{1}{2 l} y^{\prime}\left(w_{c i}-w_{c j}\right)+\frac{1}{4 l^{2}}\left(v_{c i}-v_{c j}\right)\left(w_{c i}-w_{c j}\right)\right]
\end{aligned}
$$

In Eqs. $(6 \cdot \mathbf{a}-\mathbf{c}), \Delta^{2}$ is given by

$$
\Delta^{2}=\left(u_{c i}-u_{c j}\right)^{2}+\left(v_{c i}-v_{c j}\right)^{2}+\left(w_{c i}-w_{c j}\right)^{2}
$$

The tangential linear matrix $\left[k_{c}^{T}\right]$ can be easily derived by considering perturbation of the element equation in the same way as in Ref. 17 ;

$\left[k_{c}^{T}\right]=\left[k_{c}^{E}\right]+\left[k_{c}^{L}\right]$

in which the large deflection matrix $\left[k_{c}^{L}\right]$ is given by

$$
\left[k_{c}^{L}\right]=\left[\begin{array}{rr}
{\left[k^{*}\right]} & -\left[k^{*}\right] \\
-\left[k^{*}\right] & {\left[k^{*}\right]}
\end{array}\right] ; \text { with }\left[k^{*}\right]=\left[\begin{array}{ccc}
k_{c 11}^{*} & k_{c 12}^{*} & k_{c 13}^{*} \\
& k_{c 22}^{*} & k_{c 23}^{*} \\
\text { sym. } & & k_{c 33}^{*}
\end{array}\right]
$$

where

$$
\begin{aligned}
& k_{c 11}^{*}=\frac{E A}{l}\left(\frac{d x}{d s}\right)^{3}\left[-\frac{1}{2 l}\left\{3\left(u_{c i}-u_{c j}\right)+y^{\prime}\left(v_{c i}-v_{c j}\right)\right\}+\frac{1}{4 l^{2}}\left\{3\left(u_{c i}-u_{c j}\right)^{2}+\Delta^{2}\right\}\right] \\
& k_{c 22}^{*}=\frac{E A}{l}\left(\frac{d x}{d s}\right)^{3}\left[-\frac{1}{2 l}\left\{\left(u_{c i}-u_{c j}\right)+3 y^{\prime}\left(v_{c i}-v_{c j}\right)\right\}+\frac{1}{4 l^{2}}\left\{3\left(v_{c i}-v_{c j}\right)^{2}+\Delta^{2}\right\}\right] \\
& k_{c 33}^{*}=\frac{E A}{l}\left(\frac{d x}{d s}\right)^{3}\left[-\frac{1}{2 l}\left\{\left(u_{c i}-u_{c j}\right)+y^{\prime}\left(v_{c i}-v_{c j}\right)\right\}+\frac{1}{4 l^{2}}\left\{3\left(w_{c i}-w_{c j}\right)^{2}+\Delta^{2}\right\}\right] \\
& k_{c 12}^{*}=\frac{E A}{l}\left(\frac{d x}{d s}\right)^{3}\left[-\frac{1}{2 l}\left\{y^{\prime}\left(u_{c i}-u_{c j}\right)+\left(v_{c i}-v_{c j}\right)\right\}+\frac{3}{4 l^{2}}\left(u_{c i}-u_{c j}\right)\left(v_{c i}-v_{c j}\right)\right] \\
& k_{c 13}^{*}=\frac{E A}{l}\left(\frac{d x}{d s}\right)^{3}\left[-\frac{1}{2 l}\left(w_{c i}-w_{c j}\right)+\frac{3}{4 l^{2}}\left(u_{c i}-u_{c j}\right)\left(w_{c i}-w_{c j}\right)\right] \\
& k_{c 23}^{*}=\frac{E A}{l}\left(\frac{d x}{d s}\right)^{3}\left[-\frac{y^{\prime}}{2 l}\left(w_{c i}-w_{c j}\right)+\frac{3}{4 l^{2}}\left(v_{c i}-v_{c j}\right)\left(w_{c i}-w_{c j}\right)\right] .
\end{aligned}
$$

As for the mass matrix of the cable subelement, an ordinary consistent mass matrix is assumed;

$$
\left[m_{c}\right]=\frac{m_{c}^{*} l}{6}\left[\begin{array}{rr}
2[I] & {[I]} \\
{[I]} & 2[I]
\end{array}\right]
$$

where $m_{c}^{*}$ is the mass of the cable subelement per unit length and $[I]$ is $3 \times 3$ unit matrix.

\section{(3) Hangers - massless inextensible string element}

Considering the small rotation $\Delta \psi$ of a hanger from an equilibrium state $(u, v, w)$ or $(\theta, \phi)$, shown in Fig. 3, where $u, v, w$ are three components of relative displacement in the $x, y, z$ directions, $\theta$ is the rotational angle of the hanger due to $u$, and $\phi$ is the rotational angle of $\theta$-rotated hanger due to $w$. In Fig. 3, the Lagrangian local coordinate system $\{\xi, \eta, \zeta\}$, fixed to the hanger, is defined in addition to the 
spatial fixed coordinate system $\{x, y, z\}$.

The hanger tension $t^{*}$ at the equilibrium state has the component perpendicular to the axis $\eta$ of the rotated hanger during the small rotation $\Delta \psi$. The work done by the component of the hanger tension, with the small relative displacement vector $\Delta x$ in the $\xi-\zeta$ plane, is given by

$$
\Delta W=\frac{1}{2} \frac{|\Delta x|}{h} t^{*} \cdot|\Delta x|=\frac{1}{2} \frac{t^{*}}{h}\left(\Delta \xi^{2}+\Delta \zeta^{2}\right)
$$

where $h$ is the hanger length. $\Delta \xi$ and $\Delta \zeta$ in Eq. (11), which are the components of the relative displacement vector $\Delta x$ in the $\xi$ and $\zeta$ direction, can be represented by three components $\Delta u, \Delta v, \Delta w$ of $\Delta x$ in the directions of the spacial coordinate system $\{x, y, z\}$ using

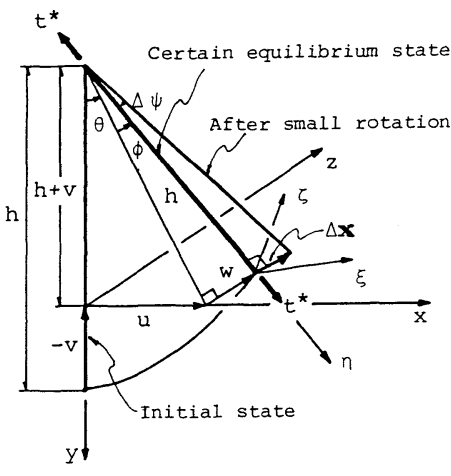

Fig. 3 Kinematics of a hanger. the ordinary transformation equation;

$$
\left\{\begin{array}{l}
\Delta \xi \\
\Delta \zeta
\end{array}\right\}=\left[\begin{array}{ccc}
\cos \theta & -\sin \theta & 0 \\
-\sin \theta \sin \phi & -\cos \theta \sin \phi & \cos \phi
\end{array}\right]\left\{\begin{array}{l}
\Delta u \\
\Delta v \\
\Delta w
\end{array}\right\}
$$

Substituting Eq. (12) into Eq. (11) and taking a variation of Eq. (11), the first variation of the incremental work is given by

$$
\delta(\Delta W)=\left[\begin{array}{lll}
\delta(\Delta u) & \delta(\Delta v) & \delta(\Delta w)
\end{array}\right]\left[k_{h}^{T}\right]\left[\begin{array}{lll}
\Delta u & \Delta v & \Delta w
\end{array}\right]^{T}
$$

Thus, the tangential stiffness matrix $\left[k_{h}^{T}\right]$ for the hanger subelement is expressed as

$$
\left[k_{h}^{T}\right]=\frac{t^{*}}{h}\left[\begin{array}{ccc}
\cos ^{2} \theta+\sin ^{2} \theta \sin ^{2} \phi & -\sin \theta \cos \theta+\sin \theta \cos \theta \sin ^{2} \phi & -\sin \theta \sin \phi \cos \phi \\
& \sin ^{2} \theta+\cos ^{2} \theta \sin ^{2} \phi & -\cos \theta \sin \phi \cos \phi \\
\text { sym. } & \cos ^{2} \phi
\end{array}\right]
$$

Referring to Fig. 3, the trigonometric functions $\cos \theta, \sin \theta, \cos \phi$, and $\sin \phi$ in Eq. (14) can be represented by the relative displacements $u, v$ and $w$ as follows:

$$
\cos \theta=\frac{h+v}{\sqrt{(h+v)^{2}+u^{2}}}, \quad \sin \theta=\frac{u}{\sqrt{(h+v)^{2}+u^{2}}}, \cos \phi=\frac{\sqrt{(h+v)^{2}+u^{2}}}{h}, \sin \phi=\frac{w}{h} \cdots \cdots(15 \cdot \mathrm{a}-\mathrm{d})
$$

Using Eq. (15) and introducing the vertical components of the hanger tension $t_{y}^{*}$, one can obtain the tangential stiffness matrix expressed in terms of the relative displacements as

$$
\left[k_{h}^{T}\right]=t_{3}^{*}\left[\begin{array}{ccc}
k_{11} & k_{12} & k_{13} \\
& k_{22} & k_{23} \\
\text { sym. } & & k_{33}
\end{array}\right]
$$

where

$$
\begin{aligned}
& k_{11}=\frac{h^{2}(h+v)^{2}+u^{2} w^{2}}{\left\{(h+v)^{2}+u^{2}\right\} h^{2}(h+v)}, \quad k_{12}=-\frac{u\left(h^{2}-w^{2}\right)}{\left\{(h+v)^{2}+u^{2}\right\} h^{2}} \\
& k_{13}=-\frac{u w}{h^{2}(h+v)}, \quad k_{22}=\frac{u^{2} h^{2}+(h+v)^{2} w^{2}}{\left\{(h+v)^{2}+u^{2}\right\} h^{2}(h+v)} \\
& k_{23}=-\frac{w}{h^{2}}, \quad k_{33}=\frac{(h+v)^{2}+u^{2}}{h^{2}(h+v)}
\end{aligned}
$$

The stiffness matrix $\left[k_{h}^{T}\right]$ in Eq. (16), which is for the relative displacement vector, should be changed into the stiffness matrix corresponding to the displacement vectors of the stiffening structure and the cable subelements. The relative displacements of the hanger are the displacements of the stiffening structure, at the corners of the cross section, relative to the displacements of the cable (Fig. 1), that is

$$
u=\left(a \theta_{v} \mp \frac{b}{2} \theta_{w}\right)-u_{c}
$$




$$
\begin{aligned}
& v=\left(v_{s} \pm \frac{b}{2} \phi_{s}\right)-v_{c} \\
& w=\left(w_{s}-a \phi_{s}\right)-w_{c}
\end{aligned}
$$

Substituting Eqs. $(18 \cdot a-c)$ into Eqs. (16), (17-a-f), the final form of the tangential stiffness matrix of the hanger corresponding to the displacement vector $\left[\begin{array}{llllllll}v_{s i} & \theta_{v i} & w_{s i} & \theta_{w i} & \phi_{s i} & u_{c i} & v_{c i} & w_{c i}\end{array}\right]$ is

$$
\left[k_{h}^{T}\right]=t_{y}^{*}\left[\begin{array}{cccc}
k_{11}^{*} & k_{12}^{*} & \cdots & k_{18}^{*} \\
& k_{22}^{*} & \cdots & k_{28}^{*} \\
& & \cdots & \vdots \\
\text { sym. } & & & k_{88}^{*}
\end{array}\right]
$$

where

$$
\begin{aligned}
& k_{11}^{*}=k_{22}, k_{12}^{*}=a k_{12}, k_{13}^{*}=k_{23}, k_{14}^{*}=\mp \frac{b}{2} k_{12}, k_{15}^{*}= \pm \frac{b}{2} k_{22}-a k_{23}, k_{16}^{*}=-k_{12}, k_{17}^{*}=-k_{22}, k_{18}^{*}=-k_{23} \text {, } \\
& k_{22}^{*}=a^{2} k_{11}, k_{23}^{*}=a k_{13}, k_{24}^{*}=\mp \frac{b}{2} a k_{11}, k_{25}^{*}= \pm \frac{a b}{2} k_{12}-a^{2} k_{13}, k_{26}^{*}=-a k_{11}, k_{27}^{*}=-a k_{12}, k_{28}^{*}=-a k_{13} \text {, } \\
& k_{33}^{*}=k_{33}, \quad k_{34}^{*}=\mp \frac{b}{2} k_{13}, \quad k_{35}^{*}= \pm \frac{b}{2} k_{23}-a k_{33}, \quad k_{36}^{*}=-k_{13}, \quad k_{37}^{*}=-k_{23}, \quad k_{38}^{*}=-k_{33} \text {, } \\
& k_{44}^{*}=\frac{b^{2}}{4} k_{11}, \quad k_{45}^{*}=-\frac{b^{2}}{4} k_{12} \pm \frac{a b}{2} k_{13}, \quad k_{46}^{*}= \pm \frac{b}{2} k_{11}, \quad k_{47}^{*}= \pm \frac{b}{2} k_{12}, \quad k_{48}^{*}= \pm \frac{b}{2} k_{13} \text {, } \\
& k_{55}^{*}=\frac{b^{2}}{4} k_{22}+a^{2} k_{33} \mp a b k_{23}, \quad k_{56}^{*}=\mp \frac{b}{2} k_{12}+a k_{13}, \quad k_{57}^{*}=\mp \frac{b}{2} k_{22}+a k_{23}, \quad k_{58}^{*}=\mp \frac{b}{2} k_{23}+a k_{33} \text {, } \\
& k_{66}^{*}=k_{11}, \quad k_{67}^{*}=k_{12}, \quad k_{68}^{*}=k_{13}, \quad k_{77}^{*}=k_{22}, \quad k_{78}^{*}=k_{23}, \quad k_{88}^{*}=k_{33}
\end{aligned}
$$

The vertical component $t_{y}^{*}$ of the hanger tension can be evaluated by considering the equilibrium of forces acting on the point where the hanger is connected with the main cable (Fig. 4); that is

$$
t_{y}^{*}=T_{n-1} \frac{h_{i-1}-h_{i}-\left(v_{c i-1}-v_{c i}\right)}{L_{n-1}}-T_{n} \frac{h_{i}-h_{i+1}-\left(v_{c i}-V_{c i+1}\right)}{L_{n}}-p_{c}^{y}
$$

where $p_{c}^{y}$ is the equivalent nodal force in the vertical direction; $T_{n}$ and $L_{n}$ are the tension and the length of the cable subelement after deformation, represented by the cable displacements as

$$
\begin{aligned}
& T_{n}=H_{w} \frac{L_{n}^{0}}{l_{n}}+E A \frac{L_{n}-L_{n}^{0}}{L_{n}^{0}} \\
& L_{n}=\sqrt{\left(l_{n}+u_{c i+1}-u_{c i}\right)^{2}+\left(h_{i}-h_{i+1}+v_{c i+1}-v_{c i}\right)^{2}+\left(w_{c i+1}-w_{c i}\right)^{2}}
\end{aligned}
$$

$L_{n}^{0}$ in Eq. (22-a) is the length of the cable subelement before deformation; $L_{n}^{0}=L_{n}\left(u_{c}=v_{c}=w_{c}=0\right)$.

\section{SUSPENSION-BRIDGE-ELEMENT MATRIX}

\section{(1) Assembly of matrices for the suspension-bridge element}

The assembly of the tangential stiffness matrix and the mass matrix for the suspension-bridge element is straightforward using the matrices for each subelements ; $\left[k_{s}\right],\left[k_{c}^{T}\right],\left[k_{h}^{T}\right],\left[m_{s}\right]$ and $\left[m_{c}\right]$, while the half of stiffness should be considered for hangers in order to use the usual direct stiffness method. The matrices corresponding to the incremental displacement vector $\left[\begin{array}{llllll}\Delta v_{s i} & \Delta \theta_{v i} & \Delta w_{s i} & \Delta \theta_{w i} & \Delta \phi_{s i} & \Delta u_{c i}^{1,2}\end{array} \quad \Delta v_{c i}^{1,2}\right.$ $\left.\Delta w_{c i}^{1,2} \quad \Delta v_{s j} \quad \Delta \theta_{v j} \quad \Delta w_{s j} \quad \Delta \theta_{w j} \quad \Delta \phi_{s j} \quad \Delta u_{c j}^{1,2} \quad \Delta v_{c j}^{1,2} \quad \Delta w_{c j}^{1,2}\right]$ can be easily obtained.

\section{(2) Reduction of DOF}

The assumption of inextensibility in the hanger causes the following kinematic relation among the relative displacements of the hanger:

$$
u^{2}+(h+v)^{2}+w^{2}=h^{2}
$$

Each relative displacement is represented in $\mathrm{Eq} .(18 \cdot \mathrm{a}-\mathrm{c})$ by the displacements of the stiffening structure and the cable. Eq. (23), then, gives a constraint condition for those displacements. Substituting Eq. $(18 \cdot a-c)$ into Eq. (23) and considering the increment of Eq. (23), the incremental displacement of cable in the vertical direction can be represented, in terms of the other incremental displacements, as 
$\Delta v_{c}=\Delta v_{s}+A_{1} \Delta \theta_{v}+A_{2} \Delta w_{s}+A_{3} \Delta \theta_{w}+A_{4} \Delta \phi_{s}+A_{5} \Delta u_{\mathrm{c}}+A_{6} \Delta w_{c}$

in which

$$
\begin{aligned}
& A_{1}=\left(a \theta_{v} \mp \frac{b}{2} \theta_{w}-u_{c}\right) a /\left(h+v_{s} \pm \frac{b}{2} \phi_{s}-v_{c}\right), A_{2}=\left(w_{s}-a \phi_{s}-w_{c}\right) /\left(h+v_{s} \pm \frac{b}{2} \phi_{s}-v_{c}\right) \cdots(25 \cdot \mathbf{a}, \mathbf{b}) \\
& A_{3}=\mp \frac{b}{2 a} A_{1}, A_{4}= \pm \frac{b}{2}-a A_{2}, A_{5}=-\frac{1}{a} A_{1}, A_{6}=-A_{2}
\end{aligned}
$$

The size of the mass matrix and stiffness matrix of the suspension-bridge element can be reduced from 22 $\times 22$ to $18 \times 18$ by the elimination of the vertical displacements of cable $\Delta v_{c i}^{1}, \Delta v_{c j}^{1}, \Delta v_{c i}^{2}$ and $\Delta v_{c j}^{2}$ from the displacement vector using Eq. (24). The computational procedure is only to add the row and column corresponding to the vertical displacements of cable, multiplied by each factor $1, A_{1}, \cdots, A_{6}$ to the other rows and columns in the matrices. It is obvious that the reduced matrices of mass and stiffness are also symmetric.

\section{EQUATION OF MOTION}

\section{(1) Stiffness matrix of tower}

It is assumed that the tower equivalent torsional and bending spring constants $k_{\alpha}$ and $k_{b}$ (in the direction of the bridge axis) can be provided at the tower top by the pre-analysis of the tower stiffness. The bending rigidity in the direction perpendicular to the bridge axis and the axial rigidity are considered large enough and accordingly their effect can be neglected. The stiffness matrix of the tower, then, is derived as

$$
\left[k_{t}\right]=\left[\begin{array}{cc}
\frac{k_{b}}{2}+\frac{k_{\alpha}}{b^{2}} & \frac{k_{b}}{2}-\frac{k_{\alpha}}{b^{2}} \\
\text { sym. } & \frac{k_{b}}{2}+\frac{k_{\alpha}}{b^{2}}
\end{array}\right]
$$

This stiffness matrix corresponds to the displacement vector $\left[\begin{array}{lll}\Delta u_{c i}^{1} & \Delta u_{c i}^{2}\end{array}\right]$.

(2) Incremental form of equation of motion

The incremental form of equation of motion for the whole structure can be constructed by the direct stiffness method using the previously derived suspension-bridge-element matrices;

$[M]\{\Delta \ddot{q}\}+\left[K^{T}(q)\right]\{\Delta q\}=\{\Delta f\}$

where $[M]$ is the mass matrix, $\left[K^{T}(q)\right]$ is the tangential stiffness matrix, $\{\Delta q\}$ is the incremental displacement vector and $\{\Delta f\}$ is the incremental force vector which is obtained easily in a standard FEM.

Eq. (27) represents the equation of motion for the small displacement vector $\{\Delta q\}$ from the certain equilibrium state $\{q\}$. In what follows, an incremental solution technique is adopted for the static analysis such as the shape analysis of the suspension bridge under construction and the analysis of deformation due to the static wind forces. That is, the following equation is solved in step by step.

$\left[K^{T}(q)\right]\{\Delta q\}=\{\Delta f\}$

Once the specific equilibrium state $\left\{q_{e}\right\}$ is obtained, one can calculate the natural frequencies and modes of the deformed suspension bridge using the linear homogeneous equation :

$[M]\{\Delta \ddot{q}\}+\left[K^{T}\left(q_{e}\right)\right]\{\Delta q\}=0$

\section{ACCURACY OF THE PROPOSED METHOD}

Numerical examples are presented to study the accuracy of the analysis developed here. The examples are based on computations for the 1/100 wind-tunnel model of the In-no-shima Oh-hashi which is one of the over-sea suspension bridges in Japan. The prototype of the bridge has a $770 \mathrm{~m}$ long center span and two $250 \mathrm{~m}$ long side spans. The geometry and the structural properties of the bridge are available in Ref. 18. The numbers of elements were taken to be 13 in the side span and 40 in the center span.

The natural oscillations, equilibrium shapes under construction and deformations due to wind forces were analyzed using the proposed method. The computed results were compared with the corresponding 


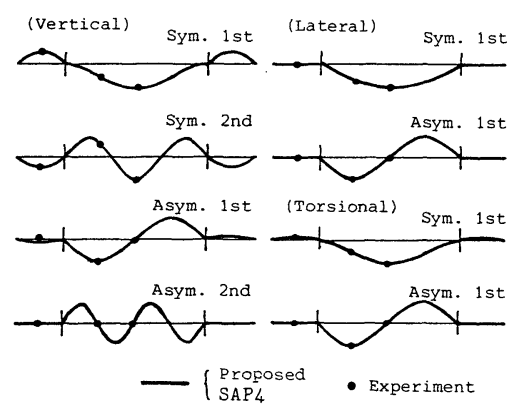

Fig. 5 Natural modes of the $1 / 100$ model of In-no-shima bridge.

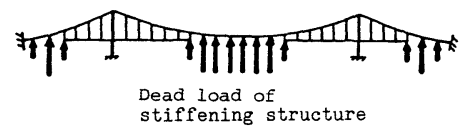

Fig. 6 Load condition for the erection shape analysis.
Table 1 Natural frequencies (in $\mathrm{Hz}$ ) of the 1/100 model of In-no-shima suspension bridge.

\begin{tabular}{|l|c|c|c|c|c|c|c|c|}
\hline & \multicolumn{4}{|c|}{ Vertical } & \multicolumn{2}{c|}{ Lateral } & \multicolumn{2}{c|}{ Torsional } \\
\cline { 2 - 10 } & Sym. 1 & Asym. 1 & Sym. 2 & Asym. 2 & Sym. 1 & Asym. 1 & Sym. 1 & Asym. 1 \\
\hline Proposed & 1.57 & 1.40 & 2.08 & 2.87 & 0.797 & 2.35 & 4.21 & 5.78 \\
\hline SAP4 $^{19)}$ & 1.56 & $\begin{array}{c}(1.16) * \\
1.43\end{array}$ & 2.06 & 2.85 & 0.793 & 2.35 & 4.09 & - \\
\hline Experiment $^{18}$ & 1.52 & 1.41 & 2.10 & 2.89 & 0.81 & 2.34 & 4.47 & 6.13 \\
\hline
\end{tabular}

* Two values for the vertical asym. 1 mode were obtained in Ref. 19.

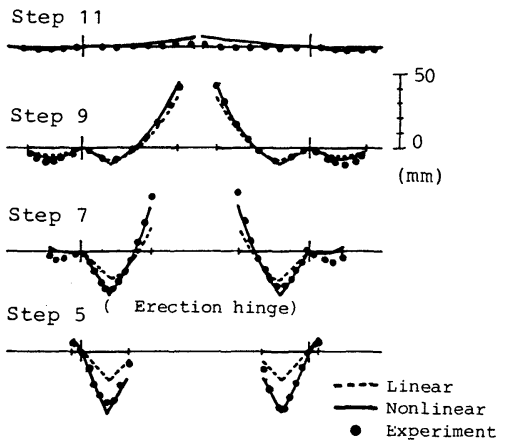

Fig. 7 Equilibrium shapes of the suspension bridge for each erection phase.

results of the wind tunnel test ${ }^{18)}$ and the results of another numerical analysis ${ }^{19)}$ in which the modified computer program of SAP 4 was utilized.

\section{(1) Natural frequencies and modes}

Natural frequencies and mode shapes of the suspension bridge can be calculated using the linear equation of motion (29) with $\left\{q_{e}\right\}=\{0\}$.

Some of the computed natural frequencies are presented in Table 1 and the associated mode shapes are shown in Fig. 5. An excellent comparison of the proposed method results can be readily observed from Table 1 and Fig. 5. It is noteworthy that the proposed method needed less than the half of SAP 4 in CPU time.

\section{( 2 ) Equilibrium shapes under construction}

The erection phases analyzed are the Step 5 (30\% erection), Step 7 (55\%), Step 9 (80\%) and Step $11(98 \%)$ in Ref. 18. Assuming the initial shape to be same as the undeformed shape in the complete phase and considering the dead load of the stiffening structure as the external force acting in the upward direction (Fig. 6), the equilibrium position of the suspension bridge under construction can be calculated by the load incremental method with Eq. (28).

Fig. 7 shows the shapes of the suspension bridge for each erection phase computed by both of the linear analysis and the nonlinear analysis. The experimentally measured values are also plotted for comparison in the figure. One can see in Fig. 7 that the calculated equilibrium shapes by the nonlinear analysis fairly coincide with the experimental results. In addition, this figure also suggests the necessity of nonlinear analysis especially for the early erection phase.

\section{(3) Deformation caused by wind forces}

The static deformations of the suspension bridge subjected to wind forces were also computed. The steady wind forces, drag, lift and moment on the stiffening structure were evaluated by using the steady-wind-force-coefficient curves shown in Ref. 20. Fig. 8 shows the lateral and vertical deflections of the stiffening structure, at the center of the center span for the complete phase (Fig. 8 (a)) and at the top of the stiffening structure for the election phase (Step 11; Fig. $8(\mathrm{~b})$ ), with respect to the wind speed. The 
results of the wind tunnel experiment and the linear analysis are also shown in Fig. 8. The good agreement of the proposed nonlinear analysis with the experimental values can be observed for every angle of wind attack $\alpha$.

\section{NONLINEAR-COUPLED OSCILLATIONS OF DEFORMED SUSPENSION BRIDGE}

\section{(1) Suspension bridge under construction}

The nonlinear-coupled free oscillations of the In-no-shima suspension bridge under construction can be calculated using Eq. (29) in which the expression for the displacement vector $\left\{q_{e}\right\}$ was derived in the section 6. ( 2 ). Fig. 9 shows the first torsional mode in which the torsional component $\Phi_{t}$ is dominant over other deflectional components $\Phi_{w s}, \Phi_{v s}$. In Fig. 9, the linear-coupled natural mode is also shown for comparison. The linear mode was obtained by the proposed analysis neglecting the erection shape of bridge $\left(q_{e}=0\right)$, that is, using the linear stiffness matrix. The corresponding natural frequencies are presented in Table 2 with the experimental results. As seen in Fig. 9 and Table 2, the nonlinear-coupled mode and the natural

(a) Complete phase
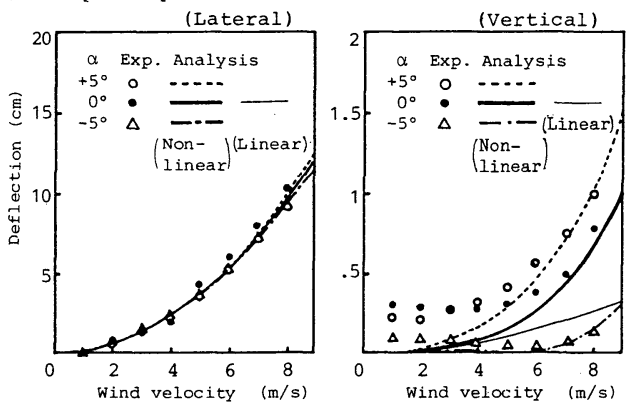

(b) Step 11

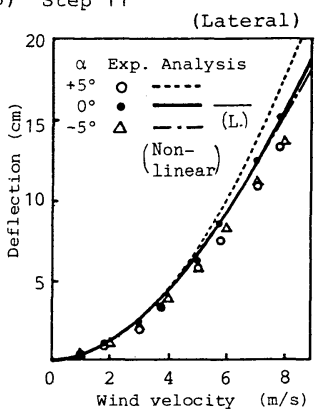

(a) Step 11

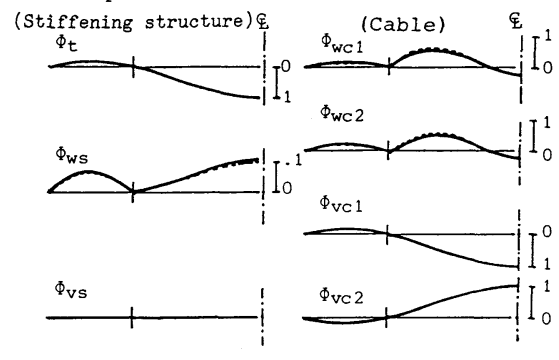

(b) Step7

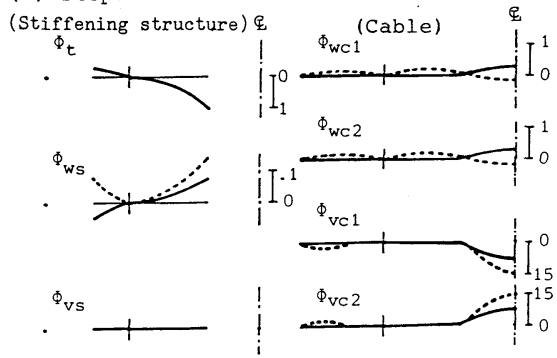

Nonlinear ...- Linear

Fig. 9 The first torsion-dominant mode of the suspension bridge under construction.

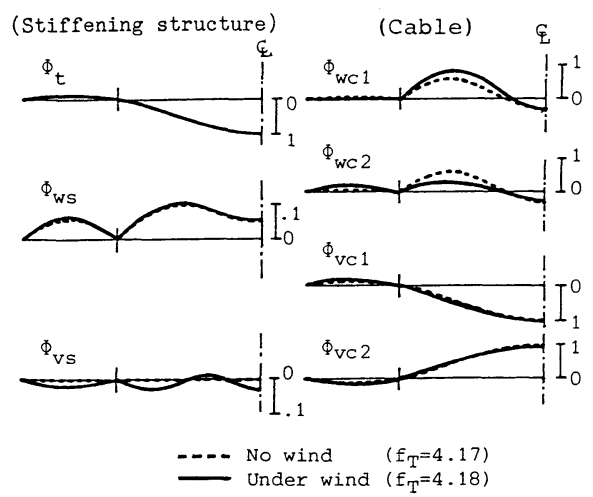

Fig. 10 The first torsion-dominant mode of the suspension bridge deformed by wind forces. 
frequency are slightly different from the linear-coupled mode and frequency for the later stage of construction (Step 11; Fig. 9(a)) whereas there are evident differences between them especially in the coupled motions of cable for the early stage (Step 7 ; Fig. 9(b)). In the early erection phase (Step 7), the huge amount of coupled motions of the free cables in the vertical direction are evident and this mode was not observed in the experiment as is shown in Table 2. This means that the first torsional mode can not exist in a practical sense because of the significant coupling of cable movements. This fact may play an important role in the evaluation of the flutter stability of suspension bridges under construction.

\section{(2) Deformed bridge under static wind loading}

The nonlinear-coupled free oscillations of the suspension bridge deformed by wind forces can be calculated in the same way using the displacement vector $\left\{q_{e}\right\}$ obtained in the section 6. ( 3 ). The calculated first torsional mode of the deformed suspension bridge under the wind with speed of $8 \mathrm{~m} / \mathrm{s}$ is shown in Fig. 10 along with the computed mode neglecting the deformation due to wind. The wind speed of $8 \mathrm{~m} / \mathrm{s}$ corresponds to $80 \mathrm{~m} / \mathrm{s}$ in the prototype. The computed natural frequencies in both cases are also presented in Fig. 10. It is confirmed from Fig. 10 that the torsional component and the corresponding natural frequency under wind loading are not so much different from those of the unloaded state, whereas the coupling between torsional, lateral, vertical and cable movements tends to be introduced by the static deformation due to wind force.

\section{CONCLUSIONS}

A method of analysis was developed for nonlinear-coupled free oscillations of suspension bridges, and the general characteristics of coupling in the first torsional mode, which is the most important mode in the wind design, were examined for the suspension bridge under construction and for the deformed bridge due to wind forces. The method, which employs a finite element technique, considers the geometrical nonlinearity caused by the large displacements of main cables and hangers.

The accuracy and efficiency of the proposed method was indicated by comparing the computed results with both the experimental ones and the calculated ones by SAP 4 program (Refs. 18 and 19). In the comparative study, the natural frequencies and modes, the equilibrium shapes under construction, and the static deformation caused by wind force were considered. The comparison shows good agreement.

The study on the characteristics of nonlinear coupling in the first torsional mode for deformed suspension bridge indicates the following aspects : vibration modes of a suspension bridge are complex due to the coupling between different components of displacement. In particular the deformed suspension bridge like the suspension bridge under construction and under strong wind has the first torsional mode accompanied by the lateral and vertical displacements of the stiffening structure and cables. As for the suspension bridge under construction, the significant coupled motions of free cables in the vertical direction are appeared especially in the early stage of construction and this fact corresponds to the experimental results that the first torsional mode could not be observed in the model experiments.

In this study, the effects of coupled motion in the first torsional mode on the aerodynamic stability are not explored. An attempt to this problem will be reported later.

\section{REFERENCES}

1) Hirai, A : STEEL BRIDGE, Gihou-Dou, 1967 (in Japanese).

2) Yamaguchi, H., Yokoyama, H. and Ito, M. : Dynamic and aerodynamic stability of a suspension bridge deformed by wind force, Proc. JSCE, No. 331, pp. 25 31, March, 1983 (in Japanese).

3) Ito, M. and Yamaguchi, H. : Effects of another wind-induced motions on torsional flutter of a suspension bridge, 12th Congress of IABSE, Final Report, pp. 697 704, 1984.

4) Yamaguchi, H., Ito, M. and Shimokawa, H. : Experimental study on dynamic response of suspension bridges in three dimensions, Annual Report of Eng. Res. Inst., Univ. of Tokyo, Vol. 40, pp. 43 48, 1981 (in Japanese).

5) Hayashi, Y. and Murata, M. : Torsional oscillation analysis of suspension bridges by a displacement method, Proc. JSCE, 
No. 258, pp. 133 144, Feb., 1977.

6) Takahashi, K, Muroi, T. and Hirano, I. : Fundamental equations of stiffened suspension bridge considering coupled deformations and its application to the system subjected to wind loads, Proc. JSCE, No.277, pp. 25 40, Sept., 1978 (in Japanese).

7) Takahashi, K. : On vertical nonlinear vibrations of suspension bridges, Proc. JSCE, No. 286, Notes, pp. 151 154, June, 1979 (in Japanese).

8) Komatsu, S. and Nishimura, N. : Effects of shear deformation on free vibrations of long span suspension bridges, Proc. JSCE, No. 323, pp. 29 41, July, 1982 (in Japanese).

9) Abdel-Ghaffar, A. M. : Free lateral vibrations of suspension bridges, Proc. ASCE, Vol. 104, No. ST3, pp. 503 525, March, 1978.

10) Abdel-Ghaffar, A. M. : Free torsional vibrations of suspension bridges, Proc. ASCE, Vol. 105, No. ST4, pp. 767 788, April, 1979.

11) Abdel-Ghaffar, A. M. : Vertical vibration analysis of suspension bridges, Proc. ASCE, Vol. 106, No. ST10, pp. 2053 2073, Oct. , 1980.

12) Abdel-Ghaffar, A. M. : Suspension bridge vibration : Continuum formulation, Proc. ASCE, Vol. 108, No. EM6, pp. 1215 1232, Dec., 1982.

13) Abdel-Ghaffar, A. M. and Rubin, L. I. : Nonlinear free vibrations of suspension bridges: Theory, Proc. ASCE, Vol. 109, No. EM1, pp. 313 329, Feb., 1983.

14) Abdel-Ghaffar, A. M. and Rubin, L. I. : Nonlinear free vibrations of suspension bridges : Application, Proc. ASCE, Vol. 109, No. EM1, pp. 330 345, Feb., 1983.

15) Ito, M. et al. : Three dimensional dynamics and aerodynamic stability of long-span suspension bridges, BEL Report, Dept. of Civil Eng., Univ. of Tokyo, March, 1981 (in Japanese).

16) Washizu, K. et al. ed. : HANDBOOK OF FINITE ELEMENT METHOD, Vol. I \&, II Baifukan, 1983 (in Japanese).

17) Henghold, W. M. and Russell, J. J. : Equilibrium and natural frequencies of cable structures (a nonlinear finite element approach) Computers \& Structures, Vol.6, pp. 267 271, 1976.

18) Ito, M. et al. : Experimental study on the aerodynamic stability of In-no-shima Bridge under construction, BEL-Report No. 81301, Dept. of Civil Eng., Univ. of Tokyo, March, 1981 (in Japanese).

19) Okimi, H. : Free oscillation analysis of suspension bridges in three dimensions, Graduation thesis, Dept. of Civil Eng., Univ. of Tokyo, March, 1982 (in Japanese).

20) Honshu-Shikoku Bridge Authority and J. V. of Kawada, Hitach, Sumi-ju and To-kotsu : Report on wind tunnel tests of In-no-shima Bridge, Oct. 1981 (in Japanese).

(Received April 7 1986) 